\title{
Utility of reprojected tomograms
}

\author{
Kenneth J. Nichols, PhD, ${ }^{\mathrm{a}}$ and Andrew Van Tosh, $\mathrm{MD}^{\mathrm{b}}$ \\ a Division of Nuclear Medicine \& Molecular Imaging, North Shore Long Island Jewish Health \\ System, Manhasset \& New Hyde Park, NY \\ ${ }^{b}$ St. Francis Hospital, Roslyn, NY
}

Received Jun 13, 2014; accepted Jun 13, 2014

doi: 10.1007/s12350-014-9948-7

Why do clinicians continue to perform gated blood pool imaging when other methods are available to assess left ventricular (LV) ejection fraction (EF)? One reason is that blood pool scanning continues to be the method favored for serial LV EF evaluations to detect cardiotoxicity caused by anthracycline therapy for several cancers in adults, ${ }^{1,2}$ and for Hodgkins disease in children. ${ }^{3}$ Echocardiography is considered inadequate for this purpose, ${ }^{3,4}$ cardiac CT delivers considerably higher radiation dose than would be desirable for serial evaluations, and cardiac MRI is not widely available. For evaluation of cardiotoxicity, simultaneous perfusion assessment is not required, because anthracyclines cause generalized myocyte failure throughout the myocardium in a uniform fashion, manifest as global hypokinesis. ${ }^{5}$

Planar blood pool scans have the advantage of simplicity and enable reproducible EF values. ${ }^{6} \mathrm{EF}$ reproducibility to within $5 \%$ has been considered a minimal requirement due to observations that a drop of more than $5 \mathrm{EF}$ points from rest to stress is a significant prognostic indicator, signaling a high likelihood of future adverse cardiac events. ${ }^{7-9}$ Building on those technical foundations, most clinicians consider a drop of more than $5 \mathrm{EF}$ points due to chemotherapy to be clinically significant, with an overall decline below LVEF of $50 \%$ to be of serious concern. ${ }^{3,10}$

Many patients undergoing chemotherapy are followed for several years after baseline assessment of cardiac function. Due to recent advances in technology, in the current nuclear imaging environment this can present referring physicians with a dilemma. They may have a long history of interpreting the implications of changes in planar blood pool EF values for patients in

\footnotetext{
Reprint requests: Kenneth J. Nichols, PhD, Division of Nuclear Medicine \& Molecular Imaging, North Shore Long Island Jewish Health System, 270-05 76th Avenue, Manhasset \& New Hyde Park, NY 11040; knichols@lij.edu; knichols@nshs.edu J Nucl Cardiol 2014;21:954-7. $1071-3581 / \$ 34.00$

Copyright (c) 2014 American Society of Nuclear Cardiology.
}

general, and have serial measurements for their own individual patients. Now they are confronted with an increasing prevalence of imaging facilities that are incapable of performing planar blood pool imaging, but instead have solid state SPECT capability, of which the referring physicians are told are superior to planar cameras. How are they going to interpret new EF results, and how will they subsequently manage their patients, when they are no longer able to obtain planar blood pool EF measurements in patients for whom they have successive planar blood pool EF values determined over many years?

In "Assessment of an Intermediate Reprojection Technique Transitioning from Planar to SPECT Radionuclide Ventriculography" in the current issue of the Journal, ${ }^{11}$ O'Doherty et al report similar EF values obtained by reprojected SPECT blood pool tomograms when compared to data in the same patients who also had conventional planar blood pool acquisitions. Why would there be any question that a reprojected tomogram would produce different $\mathrm{EF}$ values than if planar data had been acquired instead? One reason is that the reconstruction process requires multiple choices from among several available options. True maximum likelihood estimation maximization (ML-EM) iterative reconstruction algorithms involve lengthy computations and are not commercially available ${ }^{12}$; rather, accelerated iterative ordered subset estimation maximization (OSEM) algorithms are implemented instead. ${ }^{13}$ In OSEM, choices of numbers of iterations and subsets to use will influence image smoothness, and there is wide latitude in choosing these parameters. Following OSEM reconstruction, many laboratories apply additional image filters to provide an overall smoother appearance to the reconstructions. In fact, O'Doherty et $\mathrm{al}^{11}$ applied a standard Gaussian filter after OSEM reconstruction in their investigation. Recognizing this in their study design, the authors addressed this issues by analyzing three types of data: planar data, SPECT data, and reprojected SPECT data. 
The authors chose to use Anger detectors for all data collections. This was a prudent decision that helped to reduce variables, even though other investigators have reported planar blood pool LV EFs to be statistically similar to SPECT blood pool EFs obtained with a dedicated solid state CZT SPECT camera. ${ }^{14}$ Nonetheless, if O'Doherty et al had compared reprojected tomograms acquired with a solid state detector to planar data acquired with an Anger camera they would have had the additional variables of different counts, energy resolution, and spatial resolution characteristics between the two detector systems. Also, the authors chose not to apply attenuation, scatter, or resolution recovery corrections to their tomographic reconstructions, which were also appropriate choices, since these corrections could not have been applied to planar data.

This raises the question of whether results would have been different with attenuation and scatter corrections of tomograms, and perhaps future investigations will address these questions. The attenuation correction experiments reported by Sundram et al ${ }^{15}$ are intriguing in this regard, but further work in this area would likely prove revealing. Such experiments ideally would be conducted with an independent imaging reference standard of higher spatial resolution, such as CMRI or CT. In the experimental design reported here, an independent reference standard was not used, and really was not required.

So, to what extent has this carefully designed study succeeded in providing convincing evidence that EFs computed from reprojected tomograms are equivalent to planar blood pool images? The Bonferroni-corrected probability values and the Bland-Altman results indicate equivalent tests. However, these results need to be taken with a grain of salt, because the Bland-Altman limits of agreement are wide. In higher EFs, this finding would be less worrisome because those patients most likely have normal LV function. Higher EFs frequently are associated with patients with smaller end-systolic volumes, for which partial volume effects become more significant than for the larger ventricles, ${ }^{16}$ and one makes allowances for that circumstance. However, O'Doherty et al found large Bland-Altman limits of agreement, on the order of $5 \%$, not only for high EFs but also for $\mathrm{EFs}<50 \%$, and this needs to be investigated further. It would have been interesting to see logistic regression analyses of factors associated with cases for which SPECT and planar LV EFs disagreed by more than $5 \%$. To be definitive, this likely would require a larger sample size than the 47 subjects analyzed in this study, to enable analyses for subgroups of patients. Factors likely to be associated with discrepant computations include background counts and LV size. The authors list as an advantage of SPECT over planar the supposed independence of background counts, but it should be recognized that in order to segment the
LV, SPECT algorithms depend on either count gradient searches or count thresholding, the latter of which has been reported to be the more accurate approach. ${ }^{17}$ Whichever approach is followed, higher background count levels of neighboring tissue will influence LV segmentation and computed LV size. Also, for a series of progressively smaller ventricles, partial volume effects progressively decrease maximum LV signal, which also would affect LV segmentation by gradient searches or count thresholds. Consequently, it is optimistic to believe tomographic blood pool imaging can provide accurate LV volumes. Comparisons against CMRI routinely show better correlation for LV EFs than for LV volumes. ${ }^{18,19}$

Regarding advantages of SPECT blood pool imaging over planar blood pool imaging, among those listed in the accompanying article are the ability to reorient the tomograms after data have been acquired, as opposed to requiring the expertise and time needed to sequentially reposition a conventional Anger camera so as to optimize separation of LV from the RV for a best septal view. This can be a challenging task, especially when count rates are low, such as for obese patients. Also, left atrial size varies markedly and can be below the partial volume limit, and the atrium can wrap around the LV so that visual separation in a planar projection can be impossible. Consequently, the time taken to attempt reorienting a planar camera in order to visualize the left atrium sometimes will be wasted effort. Reorienting the data after tomographic acquisition should be the more successful approach. Since the tomogram contains all acquired counts there is the most information available to achieve optimal reorientation, as opposed to much fewer counts available during the acquisition process.

O'Doherty et al also present evidence for the accuracy of phase information from reprojected tomograms, although for only ten data pairs rather than for the entire patient population, as the latter would have been more convincing. While phase analysis has a very long history for planar blood pool imaging, ${ }^{20}$ SPECT enables fully $3 \mathrm{D}$ regional phase and regional $\mathrm{EF}$ information, and so can corroborate phase abnormalities seen on planar scans indicative of infarction or dyssynchrony. ${ }^{21}$ Solid state cameras have been championed as moving nuclear imaging technology forward in the realm of myocardial perfusion imaging. With their higher sensitivity, better energy resolution and better spatial resolution than conventional Anger detectors, ${ }^{22}$ they may also advance SPECT blood pool imaging. ${ }^{23}$ If the atrium is sufficiently large, 3D phase analysis may help separate atrial from ventricular counts, and this will more often succeed for solid state cameras, as these have superior spatial resolution over conventional planar Anger cameras. Consequently, with solid state detectors it should less often be problematic to separate the cardiac chambers because of partial volume effects. ${ }^{16}$ 
There is little doubt that there is a clinical role for reprojecting SPECT blood pool data. Will there also be investigatory opportunities afforded by reprojecting SPECT blood pool data? Possibly. For decades, it has been common practice to estimate background correction counts by drawing a crescent-shaped region beyond the lateral-free wall of the LV and tabulating mean counts per pixel, which then are used to subtract background counts from end-diastolic and end-systolic LV counts. Tomographic reprojection now enables exploring the validity of this approach, and quantifying the degree to which that has been an adequate approximation. Particularly with corrections for scatter, attenuation, and resolution recovery, true background counts should be obtainable, at which point accuracy of correcting planar data by sampling planar lung counts can be gauged. The accuracy of algorithms that have been used to compute LV volumes from planar data, and are widely commercially available, also can be tested against tomography,${ }^{24}$ also can be tested against tomography.

Other research areas for SPECT blood pool imaging, not confined to reprojecting tomograms, include RV as well as LV regional phase and regional EF. SPECT blood pool imaging may enable more accurate measurements of the role of right ventricular parameters in assessing prognosis, as suggested by some planar blood pool studies. ${ }^{25}$ The advantages of SPECT blood pool imaging over SPECT myocardial perfusion imaging in cases of severe hypoperfusion, and prospects for obtaining tomographic blood pool images during PET bolus injections, may well expand the way in which ${ }^{82} \mathrm{Rb}$ PET data are analyzed, and tomographic analyses of first pass data obtained during dynamic SPECT acquisitions. $^{26}$

In summary, the article by O'Doherty et al provides convincing evidence that reprojected planar gated blood pool images provide similar LV EF values as actual planar studies, though leaves some questions open as to the circumstances under which values differ by $5 \%$ or more. Insofar as tomographic data are inherently richer than planar data, further analyses of the relationships between functional parameters computed from these two types of data should provide further insight into the situations in which agreement is suboptimal, particularly if studied in conjunction with a further, third, reference standard of higher spatial resolution, such as CMRI.

\section{References}

1. Ganz WI, Sridhar KS, Ganz SS, Gonzalez R, Chakko S, Serafini A. Review of tests for monitoring doxorubicin-induced cardiomyopathy. Oncology 1996;53:461-70.

2. Feola M, Garrone O, Occelli M, Francini A, Biggi A, Visconti G, et al. Cardiotoxicity after anthracycline chemotherapy in breast carcinoma: Effects on left ventricular ejection fraction, troponin I and brain natriuretic peptide. Int J Cardiol 2011;148:194-8.

3. Tantawy AAG, Elmasry OA, Shaaban M, Toaima DN, El Shahat AM. Radionuclide ventriculography detects early anthracycline cardiotoxity in children with hodgkin lymphoma. J Pediatr Hematol Oncol 2011;33:e132-7.

4. Ewer MS, Ali MK, Mackay B, Wallace S, Valdivieso M, Legha SS, et al. A comparison of cardiac biopsy grades and ejection fraction estimations in patients receiving Adriamycin. J Clin Oncol 1984;2:112-7.

5. Pfeffer B, Tziros C, Katz RJ. Current concepts of anthracycline cardiotoxicity: Pathogenesis, diagnosis and prevention. Br J Cardiol 2009;16:85-9.

6. Massardo T, Jaimovich R, Lavados H, Gutiérrez D, Rodríguez JC, Saavedra JM, et al. Comparison of radionuclide ventriculography using SPECT and planar techniques in different cardiac conditions. Eur J Nucl Med Mol Imaging 2007;34:1735-46.

7. Jones RH, McEwan P, Newman GE, Port S, Rerych SK, Scholz $\mathrm{PM}$, et al. Accuracy of diagnosis of coronary artery disease by radionuclide management of left ventricular function during rest and exercise. Circulation 1981;64:586-601.

8. Hamad MA, van Straten AH, Schonberger JP, ter Woorst JF, de Wolf AM, Martens EJ, et al. Preoperative ejection fraction as a predictor of survival after coronary artery bypass grafting: Comparison with a matched general population. J Cardiothorac Surg 2010;5:29-36.

9. Dona M, Massi L, Settimo L, Bartolini M, Giannì G, Pupi A, et al. Prognostic implications of post-stress ejection fraction decrease detected by gated SPECT in the absence of stress-induced perfusion abnormalities. Eur J Nucl Med Mol Imaging 2011;38:485-90.

10. Mitani I, Jain D, Joska TM, Burtness B, Zaret BL. Doxorubicin cardiotoxicity: Prevention of congestive heart failure with serial cardiac function monitoring with equilibrium radionuclide angiocardiography in the current era. J Nucl Cardiol 2003;10:132-9.

11. O'Doherty J, Rojas-Fischer J, Price J, Wechalekar K. Assessment of an intermediate reprojection technique transitioning from planar to SPECT radionuclide ventriculography. J Nucl Cardiol 2014. doi:10.1007/s12350-014-9945-x.

12. Miller TR, Wallis JW. Clinically important characteristics of maximum-likelihood reconstruction. J Nucl Med 1992;33:1678-84.

13. Koch W, Hamann C, Welsch J, Popperl G, Radau PE, Tatsch K. Is iterative reconstruction an alternative to filtered backprojection in routine processing of dopamine transporter SPECT studies? J Nucl Med 2005;46:1804-11.

14. Wells RG, Marvin B, Kovalski G, Ruddy T. Planar radionuclide angiography with a dedicated cardiac SPECT camera. J Nucl Cardiol 2013;20:358-66.

15. Sundram F, Notghi A, Deakin A. Does attenuation correction of gated tomographic cardiac blood pool studies affect left ventricular volumes and ejection fraction values? Nucl Med Commun 2006;27:A47.

16. Ford PV, Chatziioannou SN, Moore WH, Dhekne RD. Overestimation of the LVEF by quantitative gated SPECT in simulated left ventricles. J Nucl Med 2001;42:454-9.

17. Daou D, Coaguila C, Benada A. Comparison of interstudy reproducibility of equilibrium electrocardiography-gated SPECT radionuclide angiography versus planar radionuclide angiography for the quantification of global left ventricular function. J Nucl Cardiol 2006;13:233-43.

18. Nichols KJ, Van Tosh A, Wang Y, Palestro CJ, Reichek N. Validation of gated blood pool SPECT regional LV function measurements. J Nucl Med 2009;50:53-60.

19. Xie BQ, Tian YQ, Zhang J, Zhao SH, Yang MF, Guo F. Evaluation of left and right ventricular ejection fraction and volumes 
from gated blood-pool SPECT in patients with dilated cardiomyopathy: Comparison with cardiac MRI. J Nucl Med 2012;53:58491.

20. Bossuyt A, Deconinck F, Demoor D, Dewilde P, Jonckheer M, Block P. Application of a temporal Fourier transform for evaluation of regional wall motion disturbances by radionuclide ventriculography at rest and at exercise. Acta Cardiol Suppl 1981;26:105-11.

21. Nichols KJ, Van Tosh A, Wang Y, De Bondt P, Palestro CJ, Reichek N. Automated detection of left ventricular dyskinesis by gated blood pool SPECT. Nucl Med Commun 2010;31:881-8.

22. Slomka PJ, Patton JA, Berman DS, Germano G. Advances in technical aspects of myocardial perfusion SPECT imaging. J Nucl Cardiol 2009; 16:255-76.

23. Jensen MM, Schmidt U, Huang C, Zerahn B. Gated tomographic radionuclide angiography using cadmium-zinc-telluride detector gamma camera; comparison to traditional gamma cameras. J Nucl Cardiol 2013;21:384-96.

24. Massardo T, Gal RA, Grenier RP, Schmidt DH, Port SC. Left ventricular volume calculation using a count-based ratio method applied to multigated radionuclide angiography. J Nucl Med 1990;31:450-6

25. van der Maas N, Braam RL, van der Zaag-Loonen HJ, Meerman J, Cozijnsen L, Scholte AJHA. Right ventricular ejection fraction measured by multigated planar equilibrium radionuclide ventriculography is an independent prognostic factor in patients with ischemic heart disease. J Nucl Cardiol 2012;19:1162-9.

26. Ben-Haim S, Murthy VL, Breault C, Allie R, Sitek A, Roth N, et al. Quantification of myocardial perfusion reserve using dynamic SPECT imaging in humans: A feasibility study. J Nucl Med 2013;54:873-9. 